The function $F_{2}$ determined by equations (6) and (7) may be found explicitly from the equation

$$
\left(x^{\prime 2}+y^{\prime 2}\right) F_{2}=y^{\prime} P-x^{\prime} Q \text {. }
$$

On expanding the second member and collecting terms, this equation becomes

$$
\begin{aligned}
\left({x^{\prime}}^{2}+y^{\prime 2}\right) F_{2}= & \left(x^{\prime} x^{\prime \prime \prime}+y^{\prime} y^{\prime \prime \prime}\right) F_{1}+\left(x^{\prime} x^{\prime \prime}+y^{\prime} y^{\prime \prime}\right) F_{1}^{\prime} \\
& -F_{x x^{\prime}}^{\prime}-F_{y y^{\prime}}^{\prime \prime}+x^{\prime}\left(F_{x^{\prime} x x}+F_{x^{\prime} y y}\right)+y^{\prime}\left(F_{y^{\prime} x x}+F_{y^{\prime} y y}\right) .
\end{aligned}
$$

Now on differentiating the identity

$$
x^{\prime} F_{x^{\prime}}+y^{\prime} F_{y^{\prime}}=F
$$

twice with respect to $x$ or $y$, the equations

$$
x^{\prime} F_{x^{\prime} x x}+y^{\prime} F_{y^{\prime} x x}=F_{x x}, \quad x^{\prime} F_{x^{\prime} y y}+y^{\prime} F_{y^{\prime} y y}=F_{y y}
$$

are obtained, so that $F_{2}$ is given by the equation

$$
\begin{aligned}
\left(x^{\prime 2}+y^{\prime 2}\right) F_{2}=\left(x^{\prime} x^{\prime \prime \prime}\right. & \left.+y^{\prime} y^{\prime \prime \prime}\right) F_{1} \\
& +\left(x^{\prime} x^{\prime \prime}+y^{\prime} y^{\prime \prime}\right) F_{1}^{\prime}+F_{x x}-F_{x x^{\prime}}^{\prime}+F_{y y}-F_{y y^{\prime}}^{\prime}
\end{aligned}
$$

In case the parameter $t$ is the length of arc, so that $x^{\prime 2}+y^{\prime 2} \equiv 1$, the function $F_{2}$ has the simpler form

$$
\begin{aligned}
& F_{2}=F_{x x}-F_{x x^{\prime}}^{\prime}+F_{y y}-F_{y y^{\prime}}^{\prime}-\left(x^{\prime \prime 2}+y^{\prime \prime 2}\right) F_{1} . \\
& \text { SheFfield Scinemific School, } \\
& \text { YaLe UnIVERSITY. }
\end{aligned}
$$

\title{
ON THE DISTANCE FROM A POINT TO A SURFACE.
}

BY PROFESSOR E. R. HEDRICK.

(Read before the Anerican Mathematical Society, September 5, 1907.)

The discussion of the extrema of the distance from a point to a surface has been made the basis for the treatment of principal radii of curvature and for the classification of points on a surface by several writers.* In this connection it is interest-

* See, e. g., Goursat, Cours d'analyse, or English translation, no. 60 ; the statements there made are correct, the example here considered falling under the case $s^{2}-r t=0$. See also Bulletin, vol. 13 , no. 9, pp. 447, 448 ; the statements of this article differ in their spirit from those of the present article, and comparisons must be made with this understanding. 
ing to notice that a variation of an example due to Peano* results in an example of a surface such that the distance from a certain point to the surface is at a minimum at a certain point of the surface for every normal plane section of the surface through that point, while the same distance is not at a minimum in general on the surface at the same point.

The surface

$$
2 x^{4}-3 x^{2} y-x^{2}-z^{2}+1=0
$$

is regular + at the point $(0,0,1)$, the normal at that point is the axis of $z$, and the square of the distance from the origin to the surface is

$$
D^{2}=x^{2}+y^{2}+z^{2}=1+\left(y-x^{2}\right)\left(y-2 x^{2}\right),
$$

so that the positive determination of $D$ in a sufficiently small circle about the origin in the $(x, y)$ plane is real and does not vanish. An extremum of $D^{2}$ therefore corresponds precisely to the same extremum of $D$. But the value of $D^{2}$ given by (2) is nothing but the Peano function

$$
F(x, y)=\left(y-x^{2}\right)\left(y-2 x^{2}\right)
$$

increased by unity. It follows that $D$ is at a minimum at $(x=0, y=0)$ along any normal section of the surface, $i$. $e_{\text {. }}$, for $y=k x$ and for $x=0$; but $D$ is not at a minimum at $(0,0)$. For these are the known properties of $F(x, y)$. That the distance from a point on the normal to each normal section is at a minimum (or at a maximum) for every normal section through the foot of the normal does not show that the same distance is at a minimum (maximum) for the surface itself.

The surface

$$
\begin{aligned}
x^{2}+z^{2}-1+3 y e^{-1 / x^{2}}-2 e^{-2 / x^{2}} & =0, \quad \text { for } x \neq 0 ; \\
x^{2}+z^{2}-1 & =0, \quad \text { for } x=0 ;
\end{aligned}
$$

is continuous and regular at the point $(0,0,1)$, the normal is the axis of $z$, and the square of the distance from the origin to the surface is

* A. Gennochi and G. Peano, Calcolo differenziale, etc., 1884, p. xxix ; German translation, Bohlmann and Schepp, 1899, p. 332.

+ That is the partial derivatives of the left-hand side of (1) exist and do not all vanish at that point. It is easy to construct and to visualize the surface (1). 


$$
\begin{aligned}
& D^{2}=1+\left(y-e^{-1 / x^{2}}\right)\left(y-2 e^{-1 / x^{2}}\right), \quad \text { when } x \neq 0 ; \\
& D^{2}=1+y^{2}, \quad \text { when } x=0 ;
\end{aligned}
$$

which is precisely the function

$$
\begin{aligned}
& \phi(x, y)=\left(y-e^{-1 / x^{2}}\right)\left(y-2 e^{-2 / x^{2}}\right), \quad \text { when } x \neq 0 ; \\
& \phi(x, y)=y^{2}, \quad \text { when } x=0 ;
\end{aligned}
$$

increased by unity; the function $\phi(x, y)$ given by $(6)$ has been studied*; it is at a minimum for any analytic curve in the $(x, y)$ plane through the point $(0,0)$; but it is not at a minimum in the region about $(0,0)$. Thus even the knowledge that the distance from a point on the normal to a surface is at a minimum at the foot of the normal for every curve cut out of the surface by an analytic cylinder through the normal, does not prove that the same distance is at a minimum for the surface itself.

Columbia, Mo.,

January 11, 1908.

\section{A GEOMETRIC REPRESENTATION OF THE GALOIS FIELD.}

BY DR. L. I. NEIKIRK.

(Read before the Chicago Section of the American Mathematical Society, March 30, 1907.)

The roots of irreducible congruences were first introduced into mathematics by Galois. $\dagger$ Various writers since then have contributed to the theory of irreducible congruences and classes of residues. $\$$ The greatest progress of recent years was made by Moore. $\$$ He proved that any finite abstract field in which division is unique is an abstract form of the Galois field; that its order is the power of a prime $p^{n}$, and that for any order it is unique, being independent of the particular irreducible congruence of degree $n$ used in defining it.

* Annals of Mathematics, vol. 8, no. 4 (July, 1907), pp. 172-174. The exact significance of "analytic," as here used, is there specified, and a more general statement of the property quoted is given.

† "Sur la théorie des nombres," Bulletin des Sciences Math. de M. Ferrussac (1830) ; also Guvres mathématiques d'Evariste Galois, GauthierVillars, Paris, 1897.

$\ddagger$ See the preface of Linear Groups by L. E. Dickson.

§ Bulletin, December, 1893 ; Chicago Congress Mathematical Papers, pp. 208-242. 